\title{
MANTENIMIENTO Y CONSERVACIÓN DE EDIFICIOS: AGENTES QUE INTERVIENEN EN LA GESTIÓN Y EXPLOTACIÓN DEL PARQUE INMOBILIARIO
}

\author{
(BUILDING MAINTENANCE: AGENTS INVOLVED IN BUILDING MANAGEMENT)
}

Justo García Navarro, Dr. Arquitecto

Profesor Titular de la Universidad Politécnica de Madrid

ESPAÑA
Fecha de recepción: 12-LX-95

\section{RESUMEN}

Durante las dos últimas décadas y especialmente en los últimos años ochenta y primeros de los noventa, la especialización profesional en el campo del mantenimiento y conservación de los edificios, asi como la implantación del concepto de "mantenimiento integral" del inmueble, se han desarrollado en España de una manera clara y progesiva. No se han realizado muchas aportaciones originales, sino que en la mayoría de los casos se han extrapolado técnicas y planteamientos ya contrastados en otros países con una mayor cultura y tradición inmobiliarias.

Este artículo -que surge como conclusiones de una conferencia impartida en el Curso de Especialización en Mantenimiento y Conservación de Edificios, celebrado por la Fundación Cultural COAM del Colegio Oficial de Arquitectos de Madrid el pasado año-, se abordan distintos agentes que intervienen en la gestión y explotación de un inmueble, y su tratamiento de manera conjunta, como base del "mantenimiento integral".

Conviene apuntar que aspectos estructurales, de acabados o de instalaciones no se analizan en profundidad, ya que fueron tratados de forma especifica en otras conferencias del citado Curso.

\section{SUMMARY}

During the last two decades, and specially at the end of the eighties and the begginning of the nineties, the professional specialization in building maintenance, and also the concept of "Integral Maintenance", were both developped in Spain. There are not many new contributions in this field. In most cases, techniques and ideas implanted and used in countries with higher real estate culture and tradition were imported.

This paper is based on the conclusions of a conference celebrated last year in the "Fundacion Cultural COAM" of the Architects Association of Madrid, and it was included in a Course of Specialization in Building Maintenance. Different agents involved in building management were discussed as well as the basis of the "Integral Maintenance"

It would be necessary to say that the structural, finishing materials and facilities aspects have not deep analyzed, because of they were separately studied in other conferences of the mentioned Course.

\section{GENERALIDADES}

\subsection{Importancia del mantenimiento}

Los inmuebles, como cualquier otro activo, necesitan durante su vida de la atención y cuidado de su propietario.
El esfuerzo no es desinteresado. Es la mejor manera de conseguir una revalorizacion continuada y el aumento de la vida útil del edificio.

Desde el ciudadano corriente, para el que la compra de una vivienda va a suponer sin duda la más importante inversión 
que realizará durante su vida y que le tendra hipotecado durante un tercio de la misma, a la gran inmobiliaria, cuyo fin es la explotación de su patrimonio, prolongar y aumentar (o cuando menos mantener) la vida y el valor de su activo debe ser el principal objetivo.

Para las empresas e inmobiliarias, con una gestión técnica y profesional, esta necesidad ya es primordial. El pequeño propietario, sin embargo, todavía está lejos de alcanzar la concienciación necesaria. Así como no dudará en pasar todas y cada una de las revisiones de su automóvil y acudirá a un taller especializado al menor síntoma de desajuste, cuando el administrador de su finca le proponga una derrama para el arreglo de alguna de las instalaciones del edificio no siempre lo entenderá como un gasto necesario e imprescindible para el mantenimiento del valor de su inversión.

\subsection{Antecedentes. Técnicas actuales}

Hasta hace pocos años los edificios, en general, se han conservado aplicando únicamente técnicas curativas o correctivas, reparando las averías una vez declaradas, con costes importantes por desperfectos colaterales y con molestias considerables para los usuarios.

Posteriormente se fueron aplicando criterios preventivos, que han tratado de minimizar los imprevistos en todos los aspectos: las revisiones periódicas de las instalaciones pueden reducir considerablemente los gastos y optimizar los rendimientos; un servicio de vigilancia o de seguridad adecuado evita robos y expolios, además de abaratar los seguros, por ejemplo.

No obstante, el mantenimiento preventivo conlleva aspectos negativos, ya que las revisiones periódicas pueden encarecerlo, además de las molestias que pueden provocar al usuario la parada de instalaciones en revisión.

Los criterios preventivos han sido mejorados con otros de tipo predictivo, que lo que hacen es medir una serie de parámetros cuya evolución va ligada al desgaste de la instalación propiamente dicha (por ejemplo, niveles de vibración, de temperatura, de presión, de carga, etc.).

Cuando la medida de estos niveles sobrepasen los límites aconsejables habrá llegado el momento de acometer la revisión y posible sustitución de piezas cuya vida efectiva haya terminado

Las últimas técnicas aplicadas van en la línea de la automatización de las instalaciones, que requieren menor mano de obra en su explotación y que ponen en funcionamiento o detienen automáticamente las instalaciones, regulando incluso su funcionamiento de acuerdo a lo previamente programado.
También se utilizan cada vez más sistemas de telemantenimiento que, como indica la propia etimología de la palabra, se basan en mantener y controlar a distancia el funcionamiento de todas las instalaciones o servicios de uno o varios edificios desde un puesto central de mando.

Finalmente, y al igual que los sistemas anteriores con una base informática, han aparecido los sistemas expertos de diagnóstico. Estos sistemas, en combinación con las técnicas antes descritas, se fundamentan en el establecimiento de las pautas de funcionamiento de las instalaciones a vigilar de tal forma que, partiendode los síntomas detectados y con una sistema de preguntas y respuestas basadas en un procedimiento lógico, se detecte con garantías la avería.

\subsection{Necesidad y rentabilidad del mantenimiento}

La vida útil de los edificios, independientemente de su uso y destino, se alarga paulatinamente. Un mantenimiento adecuado, unido a rehabilitaciones profundas que adecúan los espacios y las instalaciones a las nuevas necesidades, hacen que muchos inmuebles superen con creces el siglo de vida.

Una correcta conservación se hace, de esta forma, indispensable para la optimización de la inversión. El funcionamiento de todas y cada una de las unidades del edificio, así como una gestión profesional que controle el gasto que dicho mantenimiento origine y lo haga acorde con los beneficios que de la explotación del inmueble se deriven, son las claves.

\section{ASPECTOS PREVIOS AL MANTENIMIENTO. EL PROYECTO DE ARQUITECTURA}

\subsection{Factores a considerar desde el proyecto}

Si analizamos todos los factores que inciden en el mantenimiento de los inmuebles, podemos encontrar una serie de aspectos que, de ser tenidos en cuenta en la fase proyectual, no sólo facilitarían considerablemente la conservación posterior, sino que en muchos casos evitan complicaciones posteriores.

La tendencia actual pasa por la colaboración, en proyecto y en todas las demás fases previas a la explotación del inmueble (construcción y puesta en funcionamiento), del equipo que gestionará y gerenciará el inmueble.

Durante el proyecto, su opinión será de vital importancia para simplificar y abaratar la explotación, así como para introducir los criterios que ayuden en el "día a día" del mantenimiento.

Durante la obra, su actuación debe tomar un papel más protagonista, recopilando toda la documentación de 
operación y mantenimiento de las instalaciones, presenciando las pruebas de funcionamiento de los distintos equipos y redactando los planes de mantenimiento y administración del edificio.

Durante la puesta en funcionamiento, deberá colaborar en la supervisión de los remates y acabados, así como en la puesta a punto del funcionamiento de todas y cada una de las instalaciones.

La estrecha colaboración de los futuros gestores y gerentes del edificio y, en cualquier caso, de los propietarios o usuarios, facilitará considerablemente la labor del arquitecto y mejorará indudablemente el producto final.

\subsection{Adaptación del diseño a los distintos factores incidentes}

El trabajo conjunto antes apuntado debe aportar solidez y garantía al producto final. Sin embargo, resulta imprescindible no olvidar una serie de factores y condicionantes que, aunque obvios, vamos a tratar brevemente. No se pretende realizar ina lista exhaustiva, ni siquieran aportar soluciones; tan sólo enumerar algunos factores que darán más información al proyectista a la hora de elegir.

\subsubsection{Condiciones climáticas}

Temperaturas extremas, soleamiento, exposición al viento, humedad del ambiente, lluvia y nieve serán agentes que van a intervenir de manera protagonista en el envejecimiento y deterioro del edificio. Considerados según su magnitud desde los primeros momentos del proyecto, pueden ser manejados convenientemente y controlados de manera absoluta.

\subsubsection{Adecuada selección de los materiales}

Una vez establecidas las condiciones climáticas, y en combinación con la localización, función y características del usuario tipo del inmueble, junto a otros aspectos menos controlables -el presupuesto, por ejemplo-, se deberá estudiar cuáles son los materiales más adecuados al caso, buscando que envejecimiento y deterioro progresen en grado mínimo.

La idoneidad de los materiales debe procurarse en todos y cada uno de los que intervienen:estructurales,de cerramiento, en las instalaciones y en los recubrimientos ya que, si bien unos incidirán más durante los primeros años de vida del inmueble y le darán la categoría del acabado, otros darán el índice de su calidad transcurridos unos años $\mathrm{y}$, de no haber sido los más adecuados, provocarán actuaciones y reparaciones mucho más costosas.

\subsubsection{El color, la textura}

En zonas en las que el soleamiento y/o la polución sean importantes, las fachadas y paramentos del edificio serán los primeros que, a los pocos meses de vida, confirmarán la correcta selección del color y textura de su superficie.

La integración de ambos factores con el paisaje y el entorno es un factor que, además de favorecer la unidad medioambiental, ofrece un elevado índice de acierto en la solución adoptada.

\subsubsection{Previsión de la limpieza, mantenimiento de las instalaciones, suministros}

El conocimiento de los materiales de acabado que se introduzcan en el proyecto y su comportamiento ante la limpieza diaria debe ser otra variable más en el momento de la toma de decisiones.

Sin ánimo de ser exhaustivos, se pueden hacer algunas recomendaciones en la elección y colocación del material desde la óptica de su limpieza. Son las siguientes:

\section{a) Suelos}

Moquetas. Es el suelo más sucio. Absorben mucho polvo y son poco higiénicos

Maderas. Se limpian bien, pero sufren mucho desgaste. No aconsejables en zonas públicas o comunes.

Terrazos. Muy resistentes y sufridos.

Cerámicos. Muy resistentes. Se limpian peor que los terrazos.

Sintéticos. Sufren un deterioro progresivo y tienen mala vejez por aspecto y limpieza.

Piedras, granitos. Buena limpieza para manchas superficiales. Las profundas no salen.

b) Paredes y techos

Las pinturas no plásticas no tienen limpieza.

Las telas son muy sucias, pero se pueden limpiar con facilidad.

Las mamparas (materiales plásticos, contrachapados, etc.) tienen una buena limpieza y un buen mantenimiento.

Las calefacciones, en concreto los rädiadores, por el aire caliente que emiten, manchan mucho. 
Los aires acondicionados también manchan, yaque mueven continuamente el polvo.

Son lugares especialmente castigados aquéllos en que está permitido fumar, por el humo del tabaco.

Las persianas de interior limpian bien y se ensucian mucho menos que cortinas y estores.

\section{c) Cristales}

Hay que tener en cuenta la accesibilidad que permita su limpieza, sobre todo en los no practicables.

Previsión de góndolas de limpieza, cuando proceda.

\section{d) Fachadas}

La contaminación y la polución manchan mucho.

Debe estudiarse la forma en la que se va a mojar la fachada y cómo va a escurrir el agua de lluvia.

Se deberá considerar cómo van a envejecer, cómo se van a oxidar o cómo van a cambiar de color los materiales durante su vida.

Hay que articular, desde las fases de proyecto, los mecanismos que permitan la posterior limpieza, en función de la periodicidad prevista.

Las pintadas y "graffities" se suelen poder limpiar, pero casi siempre van a dejar "sombra". Con chorro de arena a presión la limpieza es más profunda por la característica abrasiva del método. Hay métodos preventivos que mejorarán la eliminación de la pintada cuando se produzca, y que se basan en la protección con una película continua de microceras que impermeabiliza el paramento y que, prácticamente, no se nota, no da brillo.
Una consideración similar a la de la accesibilidad a los elementos para su limpieza se debe hacer sobre el entretenimiento de las instalaciones (cambio de lámparas, medida de los equipos, etc.) y sobre la recepción y almacenamiento de los suministros (materiales, combustibles, etc.), aspectos todos ellos que deben ser considerados desde el propio proyecto.

\subsubsection{Uso y destino del inmueble}

$\mathrm{Al}$ igual que ocurre con los factores comentados, el estudio del futuro usuario y del destino y utilización que éste dará al edificio que nos ocupe serán determinantes, no sólo en el diseño, sino también en la adecuada selección de materiales, colores, texturas y, sobre todo, de los acabados.

De una correcta selección de todo ello dependerá el mantenimiento posterior del edificio y la conservación de la obra arquitectónica.

\subsection{El Manual de Mantenimiento}

Es una figura que poco a poco se va introduciendo, en gran medida gracias a las empresas que se dedican al mantenimiento integral. Al igual que con cualquier aparato de imagen y sonido, ordenadores o vehículos se entrega al usuario un manual de instrucciones, parece que en el caso de un edificio (maquinaria mucho más compleja en la que se van a interrelacionar personas, instalaciones y servicios) debería ser algo imprescindible.

La necesidad de establecer una serie de pautas sobre el mantenimiento y correcto uso del inmueble, demanda la producción de este tipo de documentos que, junto con los planos "as built" (planos definitivos que reflejan exactamente lo que se ha hecho), llegarán a ser otro documento más del proyecto en un futuro no muy lejano.
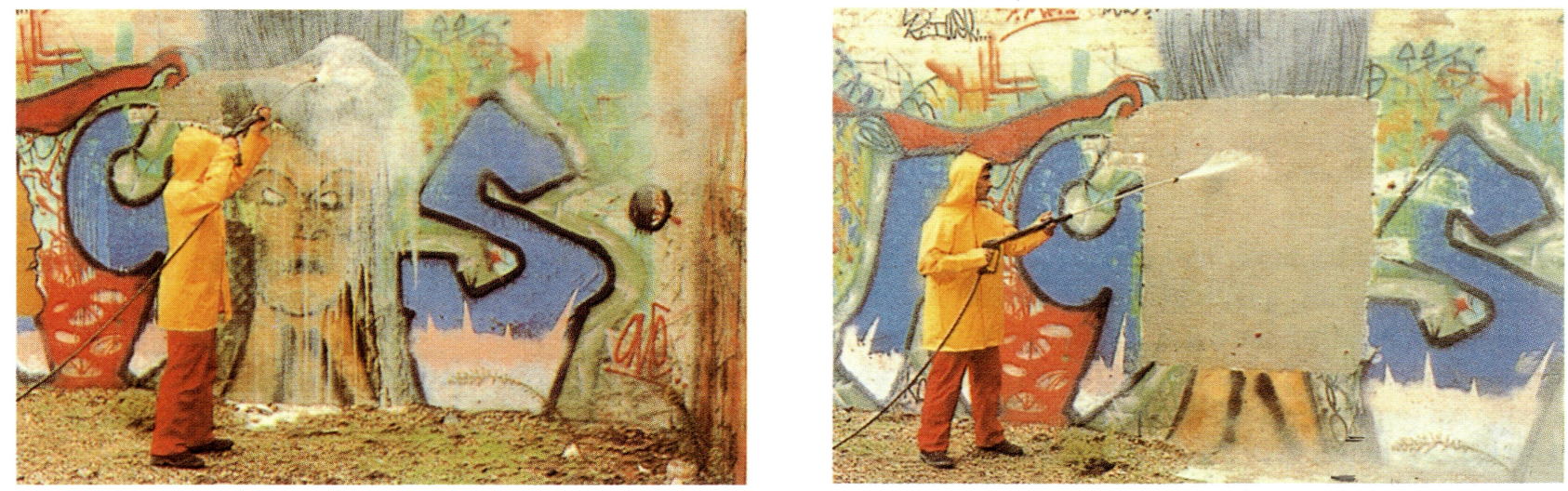

Limpieza y tratamiento de fachadas. Sistema AGS. 


\section{NECESIDADES DE SERVICIO EN LOS EDIFICIOS}

\subsection{Distintos tipos de propiedad. Distintas necesidades}

Las necesidades de servicioy mantenimiento que presentan los edificios varían en función de sus características, uso y destino.

Desde la vivienda en propiedad, unifamiliar (normalmente integrada en una comunidad constituida por la urbanización) o individual (parte de un condominio o edificio de viviendas en altura, cuyo uso principal es el residencial), pasando por locales o edificios de carácter terciario(comerciales y de oficinas), naves industriales, edificios en explotación (hoteles, aparcamientos, etc.), hasta llegar al gran edificio de oficinas o el gran centro comercial, los servicios que van demandando cada uno de ellos, aunque con un origen similar, se complejizan de muy diversa forma.

Los edificios más sencillos se suelen bastar con las figuras del administrador tradicional, que coordina, y la del porteroconserje, que concentra las funciones de control-seguridad, limpieza y mantenimiento, suplementado por casas especializadas para las instalaciones más complejas.

Los grandes inmuebles pasan a necesitar de un Director o Jefe de la infraestructura, que gestiona conjuntamente recursos humanos y medios técnicos, y que es el enlace con la empresa que gerencia y mantiene el inmueble, coordinando los distintos agentes que intervienen en la explotación.

\subsection{Agentes que intervienen en la gestión práctica}

\subsubsection{La administración. Funciones}

La Ley 49/1960, de 21 de julio, de Propiedad Horizontal, establece, en suartículo 18, las funciones del administrador de fincas. Son las siguientes:

$\left.1 .^{\circ}\right)$ Velar por el buen régimen de la casa, sus instalaciones y servicios, y hacer a estos efectos las oportunas advertencias y apercibimientos a los titulares.

$\left.2 .^{\circ}\right)$ Preparar con la debida antelación y someter a la junta el plan de gastos previsibles, proponiendo los medios necesarios para hacer frente a los mismos.

3. ${ }^{\circ}$ ) Atender a la conservación y entretenimiento de la casa, disponiendo las reparaciones ordinarias, y en cuanto a las extraordinarias, adoptar las medidas urgentes, dando inmediata cuenta a la junta, o en su caso a los propietarios.

$\left.4^{\circ}\right)$ Ejecutar los acuerdos adoptados en materia de obras y efectuar los pagos y realizar los cobros que sean procedentes.

5. ${ }^{\circ}$ Actuar, en su caso, como secretario de la junta y custodiar, a disposición de los titulares, la documentación de la comunidad.

6. ${ }^{\circ}$ Todas las demás atribuciones que se confieran por la junta.

\subsubsection{La limpieza}

Es el principal y más básico de los servicios. A pesar de su simplicidad, de él va a depender, en buena medida, el correcto funcionamiento de los demás.

Una vez finalizada la construcción del edificio, y como paso previo a la ocupación, se acomete una primera limpieza o limpieza de obra, que pretende eliminar todos los restos y residuos que durante las distintas fases del proceso constructivo se han ido produciendo, haciendo habitable y utilizable el inmueble.

Inmediatamente después de esta primera limpieza, el mantenimiento o limpieza continuada del edificio se hace indispensable, con una periodicidad marcada por el nivel de utilización de cada una de las instalaciones y por el tipo y características del inmueble.

En general, refiriéndonos a las zonas comunes de un edificio tipo de uso mixto, por elementos, y en función de la periodicidad de la limpieza, podemos distinguir:

Limpieza diaria. Aspirado, barrido y/o fregado de suelos, limpieza de polvo, eliminación de residuos (papeleras, ceniceros, etc.), limpieza de aseos.

Limpieza periódica. (Normalmente a gusto del usuario) Limpieza especial de suelos (con espuma en moquetas, abrillantado de suelos de mármol, madera o cerámicos), cristales (suele ser mensual), paredes y techos, cortinas y visillos, persianas.

Limpiezas especiales. Pulido de suelos (cada varios años), fregados especiales de suelos con máquina (en garajes, por ejemplo), limpieza de fachadas con góndola, etc.

Finalmente, conviene distinguir entre limpieza e higiene, concepto este último que engloba actuaciones singulares tales comodesinfección, desinsectación y desratización.

\subsubsection{Elmantenimiento y conservación de las instalaciones}

Resulta imprescindible para cualquier tipo de inmueble y se fundamenta, en edificios de caräcter residencial, en contratos de mantenimiento con empresas especializadas 
que en muchos casos han sido la proveedoras o suministradoras de la misma instalación.

Según aumenta la complejidad del edificio y de sus instalaciones (fontanería, saneamiento, calefacción, climatización, electricidad e iluminación, instalaciones especiales y de seguridad) la gestión y coordinación del mantenimiento se hace más difícil.

\subsubsection{La seguridad}

\subsubsection{Necesidades y concepto}

La necesidad de la seguridad se justifica en el gran incremento de riesgos que inciden sobre las Organizaciones (comunidades, empresas e instituciones) en su normal funcionamiento, y que pueden afectar tanto a personas como a bienes, producción, instalaciones e imagen.

De esta forma, se define seguridad como el conjunto de medidas, normas, recursos y actuaciones personales, que tienen como fin que la Organización funcione como tiene previsto.

\section{Se basa en tres aspectos:}

A) Recursos humanos, necesarios para el funcionamiento operativo.

B) Dispositivos técnicos, que apoyan a los recursos humanos para conferirles mayor efectividad y agilidad en muchas de sus funciones. Al mismo tiempo, disminuyen esos recursos humanos

Dentro de este tipo de dispositivos, se distinguen medios técnicos activos y pasivos. Cuando se elabora una propuesta o proyecto de seguridad, todos estos medios se agrupan en subsistemas, y se definen según la función que desarrollan dentro de la instalación. A saber:

a) Subsistema de control de accesos. Se entiende como el conjunto de medios y medidas encaminadas a la identificación de las personas, vehículos o materiales que pretenden introducirse en el interior de un recinto, impidiendo la entrada a aquéllos que no cuenten con la correspondiente autorización.

Medios activos: lectores de tarjeta, detectores de metales, detectores de explosivos, inspección por rayos $\mathrm{X}$, lectores de control de rondas.

Medios pasivos: tornos, puertas, esclusas, barreras motorizadas, barreras antivehículo, contenedores antiexplosión,etc.

b) Subsistema de protección contra la intrusión. Se define intrusión como el acto de introducirse de forma violenta, o

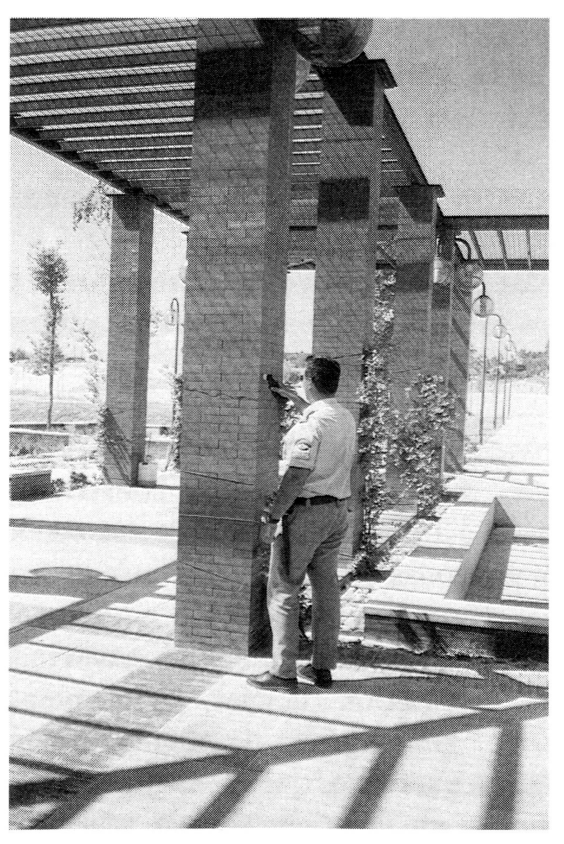

Lector de control de rondas.

utilizando artimañas, en una zona delimitada, no estando autorizado y con la intención de causar daño. Así pues, se define como sistema de protección contra la intrusión aquel conjunto de medidas y medios para detectar una intrusión no autorizada en la zona delimitada.

Medios activos.

Para la vigilancia perimétrica: circuito cerradode televisión -CCTV-

Para la detección de intrusión perimetral: soportados, autosoportados, enterrados.

Para la detección de intrusión periférica: puntuales, lineales, superficiales, volumétricos.

Medios pasivos: alambradas y concertinas, cerramientos y vallados, muros y tabiques, barreras de detención, puertas fuertes, blindadas y acorazadas, rejas y empalizadas, cierres y persianas, vidrios de seguridad, etc.

c) Subsistema de protección contra la agresión. Se entiende por protección contra la agresión todos aquellos medios tanto de carácter activo como pasivo, encaminados a impedir un ataque directo a las personas o bienes objeto de la protección.

Medios activos: sistemas de transmisión de alarma, pulsadores y accesorios de alarma y antiatraco, sistemas de fotografía y grabación, barreras antivehículo, persianas de cierre automático (antiatraco), etc.

Medios pasivos: puertas acorazadas, puertas blindadas, puertas fuertes, cámaras acorazadas, cajas fuertes, cabinas 
blindadas, blindajes (opacos, translúcidos o transparentes, etc.).

d) Subsistema de protección contra el fuego. Se define como protección contra el fuego la acción de defender, con las medidas y medios necesarios, a las personas y los bienes contra los daños que puedan derivarse de un incendio, incluyendo su prevención o acción de tomar dichas medidas y medios para evitar que se produzca.

Medios activos: detección automática de incendios, instalaciones de alarma (pulsadores de alarma, instalación de alerta, instalación de megafonía), instalaciones de emergencia (alumbrado de emergencia, alumbrado de señalización, presurización de áreas de independencia, accionamiento de puertas cortafuego, ventilación de vestíbulos de independencia), sistemas para la extinción de incendios, sistemas automáticos de extinción (rociadores automáticos de agua, difusores de polvo extintor, difusores de agentes gaseosos $-\mathrm{CO}_{2}$, halón, etc.-), extintores móviles, red de bocas de incendios equipadas, red de hidrantes de incendios, columna seca.

Medios pasivos: sectorización y compartimentación de las áreas de riesgo, salidas de emergencia, escaleras de evacuación, señalización de las vías de evacuación y medios de lucha contra incendios, elementos cortafuegos (puertas, vidrios), sellado de canalizaciones eléctricas.

e) Subsistema de comunicaciones y centralización de alarmas. Mediante un Centro Operativo de Seguridad -COS-, cuya finalidad es la de centralizar todas las señales de los subsistemas descritos anteriormente, se vigilan todas las operaciones relativas a la seguridad de la Organización, además de visionar las imágenes procedentes del CCTV y telemandar las cámaras de TV motorizadas. Deberá contar con una autonomía de funcionamiento ante un posible fallo del suministro eléctrico, sin alterar el funcionamiento de ninguno de los subsistemas.

Deberá disponer, además, de un sistema radiotelefónico, configurado por una estación base en el COS y las estaciones móviles de tipo portátil, necesarias para ser utilizadas por el Departamento de Seguridad y el personal de vigilancia en sus desplazamientos por la Organización.

Medios necesarios:

Centro Operativo de Seguridad. Lugar físico ubicado en la Organización y con unas características acordes con la función que debe cumplir (es decir, teniendo en cuenta que allí debe ubicarse un armero y la centralita de alarmas, por lo que el recinto irá protegido y blindado).

Consola de vigilancia y control, que contendrá la Central mixta de alarmas de fuego, agresión e intrusión, y que estará compuesta por pantalla, teclado, impresora,

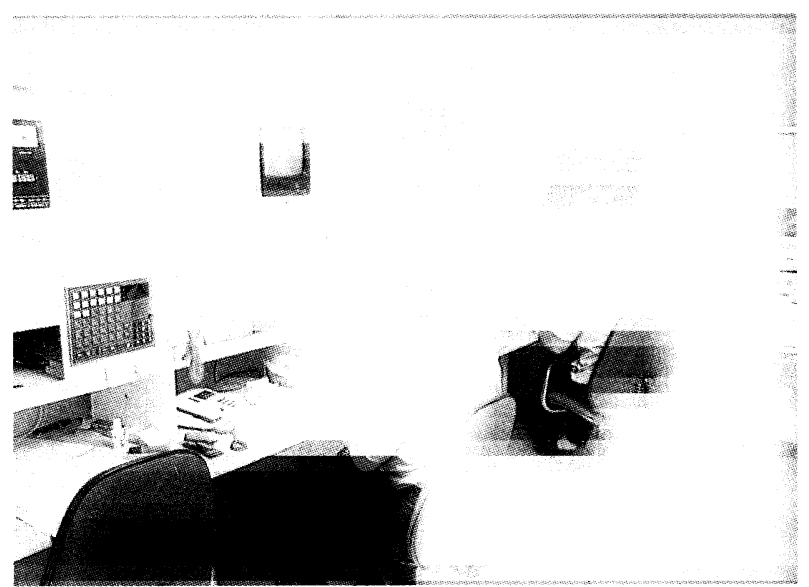

Centro Operativo de Seguridad.

ordenador personal, panel de entrada de alarmas, panel de salidas, fuente de alimentación, central de intercomunicación, central de megafonía, emisora de radio, teléfono interior/exterior, control de rondas, matriz de conmutación de vídeo, monitores de CCTV, videograbador reproductor, sistema de alimentación ininterrumpida, cuadro eléctrico de alimentación.

C) Medidas organizativas, que coordinan y conjuntan los medios humanos con los técnicos.

\subsubsection{El proyecto de seguridad. La seguridad integral}

"El nivel de seguridad de una Organización lo mide el punto más débil de la misma".

Éste es el principio del que se debe partir a la hora de abordar un proyecto de seguridad. Dicho proyecto debe seguir un esquema como el que sigue:

Estudio y análisis de riesgos

Elaboración de soluciones para problemas determinados. Diseño de organizaciones.

Definición de puestos de trabajo en el Area de Seguridad. Perfeccionamiento de sistemas.

Programas de formación, selección y capacitación del personal.

Programas de Seguridad, organización y planificación de la misma.

Protección de la Información.

Coordinación con:

Fuerzas de la Seguridad del Estado.

Cuerpos de Bomberos.

Servicios de Protección Civil. 
Las funciones o necesidades básicas del proyecto para alcanzar la finalidad de protección del inmueble son:

Disuasión, o labor preventiva del sistema de vigilancia; en ella intervendrán, para obstaculizar y retardar las posibles amenazas, todos los recursos humanos y técnicos tanto activos como pasivos.

Detección, por la que se identifica y localiza cualquier intento de intrusión y avisa de que se intenta o se está produciendo una alarma o vulneración del inmueble, o de hechos que pueden producirse con el consiguiente riesgo para las personas y bienes protegidos.

Valoración, o reconocimientode la situación, identificación de la agresión, el peligro, el accidente, evaluando lo que está ocurriendo y discriminando las alarmas falsas de las reales y el alcance de las mismas

En función de esta evaluación se tomaran las medidas necesarias.

Reacción, llevada a cabo con los recursos humanos disponibles. Se intentará neutralizar el peligro y si la agresión es de cierta envergadura, se solicitarán ayudas exteriores(Fuerzas y Cuerpos de Seguridad, bomberos, etc.).

\subsubsection{Otros servicios}

Es necesario tener en cuenta que de la complejidad, concepto y modernidad del inmueble depende el número y categoría de sus servicios e instalaciones.

De esta forma, y además de los ya comentados agentes básicos que intervienen en la explotación del edificio (administración, limpieza, mantenimiento de instalaciones y seguridad) conviene citar cuando menos la existencia de otro tipo de servicios e instalaciones (jardines, instalaciones deportivas o de ocio, etc.), en cuyo mantenimiento y gestión se aplican criterios como los ya desarrollados y que, por su carácter no básico y suntuario, encarecen sensiblemente el mantenimiento y conservación del inmueble, aunque simultáneamente lo revalorizan.

\section{LAS NUEVAS FIGURAS: EL MANTENIMIENTO INTEGRAL}

\subsection{EI mantenimiento integral como alternativa}

Lacoordinación y gestión conjunta de los agentes analizados en el punto anterior se hace cada vez más compleja, tanto para el gran edificio de oficinas, como parauna urbanización de carácter residencial o un condominio.
Esa dificultad ha provocado la aparición de empresas dedicadas al mantenimiento integral de edificios que aportan como principal ventaja el hecho de constituirse en interlocutor único para el cliente.

Aunque hasta la fecha la oferta de mantenimiento integral suele comprender los servicios de limpieza, mantenimiento de instalaciones y seguridad, la tendencia a concentrar la gestión de los servicios hace suponer que, a corto plazo, la administración se integre también de forma habitual dentro del mismo paquete, de forma que la prestación sea total y única.

\subsection{La gestión de la infraestructura o "facilities management"}

Es el conjunto de técnicas necesarias para la adecuada dirección de los recursos humanos y materiales en un contrato de mantenimiento integral.

El Gerente de la infraestructura del edificio o "Facilities Manager" es el director del entramado formado por el personal propio y subcontratado que interviene en la prestación del mantenimiento integral.

Sus funciones y objetivos serán:

- Asegurar el funcionamiento y el rendimiento nominal de las instalaciones y sistemas.

- Aumento de la disponibilidad de los servicios y de las prestaciones.

- Disminución del coste de mantenimiento, consiguiendo una explotación económica.

- Detección y eliminación de puntos débiles.

- Reducción de averías imprevistas por la detección a tiempo de posibles daños mediante programas preventivos.

- Prolongación de la vida útil de las diferentes instalaciones y reducción, por tanto, de las inversiones de reemplazo.

- Reducción del coste de explotación, al mantener el sistema en su punto óptimo de funcionamiento.

\section{OTROS ASPECTOS A CONSIDERAR}

\subsection{El seguro y los inmuebles. Generalidades}

En este último capítulo se pretende aportar una sucinta información en relación con el segure. Por ello, y para mayor abundamiento sobre los aspectos que se van tratar, 
la referencia será la Ley 50/1980, de 8 de octubre, de Contrato de Seguro, y la nueva ley de Ordenación y Supervisión de los Seguros Privados, de inminente aprobación.

Una primera aclaración, previaa cualquier otro comentario: el seguro no es una forma de mantener, no es un contrato de mantenimiento. El seguro, por definición, no puede ser objeto de lucro y tan sólo tendrá efecto cuando se produzca el accidente o siniestro, que es aquello que resulta imprevisto, súbito y ajeno a la voluntad del asegurado.

De esta forma, los contratos de mantenimiento, de conservación y de seguridad, y el contrato de seguro del inmueble siguen líneas paralelas, y sólo se relacionan por el hecho de que cuanto mayores y mejores sean las prestaciones de los primeros, más se abaratarán las primas del segundo.

Del mismo modo y en líneas generales, dichas prestaciones sobre conservación y entretenimiento de las instalaciones serán responsabilidad de los mantenedores, y sólo cuando se produzca el siniestro (imprevisto, súbito y no deseado) se obtendrá la correspondiente indemnización de la aseguradora.

Es importante igualmente conocer el carácter de adhesión del contrato de seguro. Es decir, que no es un contrato que se negocia, sino que ante una póliza que consta de unas condiciones generales (aprobadas por la Dirección General de Seguros), unas condiciones particulares (que definen propiamente el riesgo que cubrirá la póliza) y unas condiciones especiales (que pueden existir o no), el asegurado se adhiere y acepta el conjunto de condiciones establecidas.

Se debe indicar que en el caso de que el inmueble asegurado tenga alguna hipoteca, deberá figurar una cláusula de beneficiario a favor de la entidad de crédito.

\subsection{Los seguros de inmuebles}

Hasta finales de los años sesenta, los seguros de inmuebles que se contrataban cubrian únicamente responsabilidad civil e incendio. Desde entonces, y tomando un protagonismo progresivo, el seguro multirriesgo y el todo riesgo han sustituido a los antes citados.

Muy sucintamente, el multirriesgo combina distintos tipos de riesgos individuales, detallando en la póliza, nominadamente, todos y cada uno de los riesgos cubiertos. Es el más común en la actualidad, sobre todo en comunidades.

El todo riesgo sigue un sistema inverso, en cuanto que cubre todo lo que no está específicamente excluido en la póliza. Normalmente va acompañado de franquicias altas, es decir, cantidades expresamente pactadas que se deducirán de la indemnización que corresponda en cada siniestro, que sirven para abaratar sensiblemente las primas.

La duración más frecuente en este tipo de seguros es anual, renovable tácitamente y cancelable con dos meses de anticipación sobre el vencimiento. Suele ser recomendable incluir una cláusula de revalorización automática (con el I.P.C.) que actualiza anualmente el capital asegurado.

Con relación al capital, conviene recordar que sólo se asegurará el edificio, y nunca el suelo sobre el que éste se ubica. Además, es el propio tomador de la póliza el que valora el capital asegurable. Los peritos tan solo intervendrán de producirse el siniestro, comprobando los términos del contrato y estableciendo la indemnización.

A este respecto, debemos considerar que debe siempre asegurarse por el valor real del inmueble, ya que en el caso de que sobrevaloremos se incurrirá en sobreseguro, y de producirse un siniestro la indemnización correspondería al valor real y nunca a la cifra asegurada (se recuerda que el seguro no puede ser objeto de lucro); en el caso de que el inmueble se haya infravalorado al establecer el capital asegurado se incurre en infraseguro, y el asegurado se convierte en coasegurador del riesgo con la compañia aseguradora, que siempre pagaría proporcionalmente a lo asegurado.

También interesa definir en este momento dos conceptos que intervendrán a la hora de contratar en forma de alternativa: valor venal, por el que el edificio se asegura por su valor en el momento inmediatamente anterior al del siniestro, y valor de nuevo, por el que el edificio se asegura para que en caso de siniestro sea reconstruido nuevamente (normalmente no se admiten valores que superen en un $30 \%$ el valor venal).

Es importante indicar que en el caso de que se produzca una agravación del riesgo asegurado debe comunicarse de inmediato a la compañía, que en su caso revisará las condiciones del contrato y la prima.

Asimismo, en el caso de que haya concurrencia de seguros, siempre se mantendrá el principio de que el seguro no puede ser objeto de lucro y en el caso de que se produzca el siniestro, cada compañía pagará proporcionalmente al capital que tenga asegurado.

Finalmente, dos recomendaciones. La primera, en relación con los conceptos continente (lo que es fijo en el edificio) y contenido (todo lo demás). El continente es lo cubierto por el seguro que contrata la comunidad de propietarios, y el contenido es lo que contrata a título particular cada propietario individual. Con el fin de que no haya problemas en la delimitación de lo que es continente y lo que es 
contenido, es recomendable que se suscriban ambas pólizas con la misma compañía aseguradora.

La segunda, se refiere al agente de seguros. Los agentes pueden ser afectos (vinculados a una única compañía) o libres (pueden ofrecer contratos con cualquier aseguradora). El hecho de contratar a través de un agente no supone encarecimiento alguno de la prima, por lo que el trato con un agente libre debe suponer como ventaja adicional que nos serán ofertados los mejores productos del mercado para cada tipo de seguro, productos que habrán sido convenientemente estudiados por el profesional. Ello no sólo nos dará la mejor posibilidad de elección a la hora de contratar, sino que cuando se produzca el siniestro será el propio agente el que se ocupe de que se nos atienda e indemnice debidamente.

\section{publicación del IETCC/CSIC}

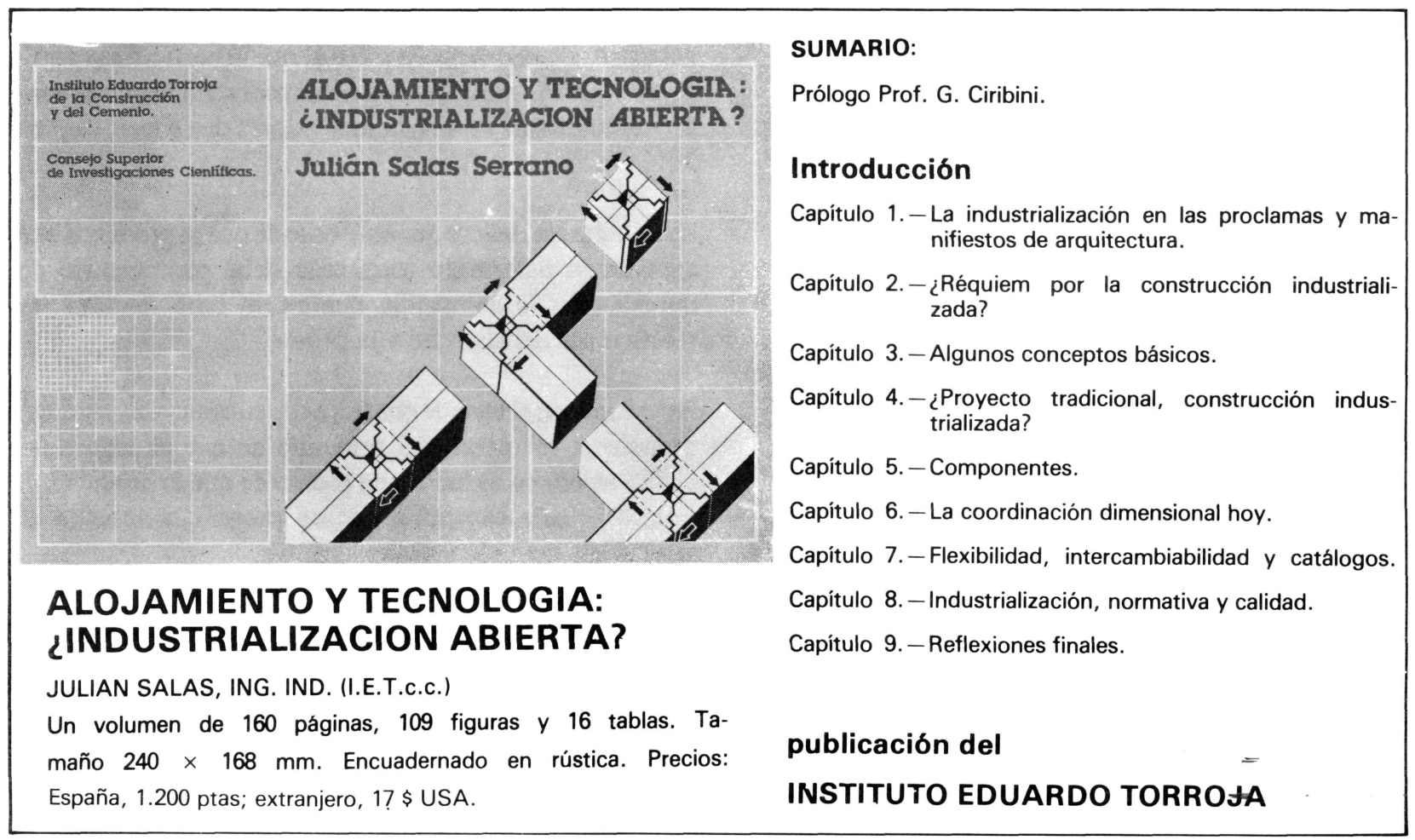

